\title{
Estimation des apports de produits phytosanitaires à l'échelle de bassins d'alimentation
}

\author{
L Heydel $^{1,2}$, M Benoît ${ }^{1}$, M Schiavon ${ }^{2 *}$ \\ ' Inra, BP 35, F-8850I Mirecourt \\ 'Ensaia, BP 172, F-54505 Vandeure-lès-Nancy, France
}

(Reçu le 2 avril 1996 : accepté le 4 décembre 1996)

\begin{abstract}
Summary - Estimating the amount of pesticides applied on watersheds. Pesticide input parameters are required for using the main models which have been proposed to describe the fate of pesticides. We suggest a method to evaluate the input of agrochemicals on an agricultural watershed. First, land use was estimated using aerial photographs, fields observations, and data from the French National Agricultural Census (RGA). Second, the input of agrochemicals on each cropping was estimated, since 1970, from a survey at the farm level. These data were confirmed by local marketing technicians. Finally, the total quantity of agrochemicals was computed as the product of the input of agrochemicals on each cropping and the surface area of each type of land use. Provided that a suitable evaluation procedure of land use is used, this method should be applied to other types of agricultural pollutants.
\end{abstract}

land use / agrochemicals / survey / aerial photograph / French National Agricultural Census

Résumé - Les apports de produits phytosanitaires pour des bassins d'alimentation dont la surface totale atteint le millier d'hectares sont évalués à l'aide de la surface occupée par chaque culture et des doses moyennes annuelles de matières actives apportées à l'hectare par culture. La reconstitution de l'assolement a été réalisée à l'aide de photographies aériennes, d'enquêtes de terrain et des données statistiques des Recensements généraux agricoles (RGA). Pour les années où elles ont pu être utilisées simultanément, les différentes méthodes donnent des résultats analogues. Leur utilisation permet de reconstituer l'assolement de 1955 à 1993. Les quantités de produits phytosanitaires apportées sur chaque culture ont été estimées par des enquêtes réalisées auprès de l'ensemble des agriculteurs exploitant le territoire étudié. Ces données ont été confirmées par les vendeurs locaux de produits phytosanitaires. Cette enquête auprès de l'ensemble des agriculteurs exploitant le site permet l'estimation des quantités de produits phytosanitaires utilisés depuis 1970 sur le maïs et depuis 1989 sur les céréales. Une telle méthode devrait être transposable à l'ensemble des polluants d'origine agricole, son extension à d'autres régions étant toutefois subordonnée à la qualité des méthodes d'évaluation des assolements des bassins d'alimentation.

assolement / pratiques phytosanitaires agricoles / enquête / photographie aérienne / recensement général agricole

* Correspondance et tirés à part.

Tél : (33) 0383595845 ; fax : (33) 0383595916 ; courriel : schiavon@ensaia.u.nancy.fr 


\section{INTRODUCTION}

Les produits phytosanitaires appliqués sur les parties aériennes des végétaux ou au sol peuvent être entrainés par les eaux superficielles ou souterraines par ruissellement et par infiltration. Par exemple, l'atrazine et la simazine, herbicides couramment employés sur le maïs, sont retrouvées dans les rivières (Hörmann et al, 1979 ; Goolsby et al, 1991 ; Maupas et Gillet, 1992) mais aussi dans les eaux souterraines (Pionke et Glotfelty, 1989 ; Domagalski et Dubrovsky, 1992). Maupas et Gillet (1992), après analyse de prélèvements effectués en rivière, décèlent la présence dans l'eau de molécules appartenant à la famille des organochlorés, des urées substituées, des aryloxyacides ou même des dinitrophénols.

Dans le cas exposé ici, les transferts de produits phytosanitaires sont étudiés pour des bassins d'alimentation dont les eaux de sources présentent des traces d'atrazine et de ses métabolites (Heydel et al, 1996).

Les huit bassins d'alimentation retenus, d'une centaine d'hectares chacun, forment deux plateaux calcaires isolés hydrauliquement des plaines qui les entourent (Salou, 1992), les plateaux du haut Saintois. Ils sont situés en Lorraine à $50 \mathrm{~km}$ au sud de Nancy, à la limite des régions du Xaintois, du Vermois et du plateau de Haye. Afin d'apporter des éléments permettant de comprendre cette pollution nous insistons, comme Mouvet et al (1992), sur la nécessité de compléter les études de transfert en laboratoire par des travaux de terrain tenant compte des données pédoclimatiques ainsi que de la nature et des quantités de produits phytosanitaires appliqués (Heydel et al, 1994).

Il s'agit ici de quantifier, sur plusieurs années, l'ensemble des matières actives appliquées sur les parcelles en maïs et en céréales des bassins d'alimentation dont les sources présentent des traces de xénobiotiques. La connaissance de ces valeurs présente un intérêt non négligeable puisqu'elles constituent l'une des variables de forçage des modèles de transfert de résidus de produits phytosanitaires (modèle Chemicals, Runoff, and Erosion from Agricultural Management systems (Knisel, 1980) et modèle Groundwater Loading Effect of Agricultural Management Systems (Knisel, 1993)). Les quantités de matières actives appliquées sur l'ensemble des parcelles d'un bassin d'alimentation sont obtenues en faisant le produit des évaluations d'une part des doses moyennes annuelles de matières actives épandues (par hectare et par culture) par les évaluations des surfaces occupées par chaque culture.

\section{MATÉRIEL ET MÉTHODES}

\section{Évolution de la surface occupée par chaque culture}

Quatre méthodes ont été utilisées pour déterminer l'occupation du sol et son évolution. La première consiste en l'observation visuelle directe des couverts végétaux. À bord d'un véhicule, l'observateur note les cultures de chaque parcelle sur un transparent rhodoïd fixé sur une photographie aérienne récente. Si cette technique ne permet pas de connaître l'occupation du sol des années antérieures, elle donne rapidement et avec précision l'occupation du sol et le parcellaire d'une région donnée pour l'année en cours. Cette méthode nécessite moins d'une journée d'observation pour couvrir le millier d'hectares des plateaux du Haut Saintois, dont l'occupation du sol est déterminée annuellement selon cette méthode depuis 1990.

La deuxième méthode repose sur l'interprétation de photographies aériennes. Les cultures sont identifiées à l'aide des teintes, des structures et des textures caractéristiques de chaque culture à une date donnée (Girard et Girard, 1989). La date de la prise de vue joue un très grand rôle dans la différenciation des espèces végétales. Seules des images aériennes prises du mois de juin au mois d'août permettent dans cette région, de distinguer le maìs et les céréales à petits grains (désignées par la suite par céréales). Nous avons donc interprété les missions aériennes de juillet 1979 et 1988 ainsi que celles des années 1987,1993 et 1994 prises au mois d'août. L'identification se fait principalement à l'aide de la teinte. Celle-ci est d'autant plus foncée que les végétaux sont riches en chlorophylle. Aux mois de juillet et août, le maïs est en pleine croissance, il est riche en chlorophylle, alors que les céréales à paille ont déjà commencé à jaunir. Sur les photographies aériennes estivales, le maïs se reconnaît donc à sa teinte foncée, alors que les céréales sont claires. Des critères de structure facilitent l'identification des céréales à paille. Par exemple, les traces des passages de roues apparaissent sous la forme de lignes parallèles un peu plus claires tant que la parcelle n'est pas moissonnée. Après la moisson on peut occasionnellement observer des bottes de paille. Le maïs se distingue des autres couverts chlorophylliens par sa texture marbrée.

Les résultats obtenus par ces deux méthodes ont été cartographiés à l'aide d'un système d'information géographique (Arc Info), afin d'obtenir des cartes de l'occupation du sol à diverses dates.

Les méthodes de télédétection sont aussi adaptées à l'identification et à l'inventaire cartographié des cultures, à condition que les parcelles ne soient pas d'une taille trop réduite (inférieure à deux hectares) (Girard et Girard, 1989). Dans notre cas, la majorité des parcelles ne dépassent pas un hectare de surface ; cette méthode est donc inutilisable.

La troisième méthode consiste à utiliser les données statistiques des Recensements généraux agricoles (RGA) pour estimer l'occupation du sol. Ainsi Morange et Salleron (1982) établissent l'inventaire des 
modes d'occupation du sol sur le bassin versant de la Moselle $\left(6850 \mathrm{~km}^{2}\right)$. Ces données ont l'avantage d'être disponibles pour le territoire étudié sur les quatre dernières décennies (1955 à 1988). Mais d'après Gaury (1992), les données des RGA ne seraient pas utilisables pour déterminer l'occupation du sol des bassins versants de quelques centaines d'hectares. En effet, les renseignements qui concernent moins de trois exploitations tombent sous le sceau du secret statistique. De plus, les superficies communales renseignées par ces données se rapportent aux surfaces des exploitations qui ont leur siège dans la commune, quelle que soit la localisation des parcelles à l'intérieur ou à l'extérieur du finage communal. C'est pourquoi, nous proposons, d'une part, d'étudier les données brutes du RGA et d'autre part de les traiter en tenant compte des caractéristiques du territoire étudié.

La quatrième méthode a donc consisté à apporter des modifications au RGA en considérant les particularités suivantes.

Les huit bassins d'alimentation qui nous intéressent sont situés sur deux plateaux calcaires, dont les sols limonosableux profonds, se travaillent plus facilement que les sols argileux des plaines voisines. Dix communes (trente agriculteurs) se partagent de façon inégale les terres des plateaux. En effet, les agriculteurs de deux d'entre elles en exploitent plus de $60 \%$, et ont l'ensemble de leurs terres labourables sur ces plateaux, ainsi que des prairies. Les exploitants des autres communes, n'ayant pas suffisamment de terres sur les plateaux, n'y installent pas de prairies mais uniquement des cultures assolées. Ils complètent les besoins en fourrage (maïs ensilage) de leurs animaux en installant des cultures assolées dans les plaines.

En conséquence, les données statistiques des RGA des communes ont été traitées de deux manières, selon que ces dernières possèdent ou non l'ensemble de leurs terres labourables sur les plateaux. De plus, on suppose que la SAU exploitée par chaque commune sur les plateaux ne varie pas au cours du temps. On considère qu'elles ont l'ensemble de leurs terres labourables sur les plateaux si la surface exploitée sur ces derniers par l'ensemble des agriculteurs d'une commune est supérieure à leur surface de terre labourable, soit

$$
\mathrm{T} p i>\mathrm{T} l i
$$

avec Tpi: surface de terre exploitée sur les plateaux par les agriculteurs de la commune $i$;

$\mathrm{T} / i$ : surface de terre labourable exploitée par les agriculteurs de la commune $i$ (surface agricole utile moins surface toujours en herbe).

Pour les communes ayant l'ensemble de leurs terres labourables sur les plateaux, on suppose que l'ensemble des céréales ainsi que le maîs sont cultivés sur les plateaux, soit :

$$
\mathrm{M} p i=\mathrm{M} i \text { et } \mathrm{C} p i=\mathrm{C} i,
$$

avec $\mathrm{Mi}(\mathrm{C} i)$ : surface exploitée en maïs (en céréales) par les agriculteurs de la commune $i$; Mpi (Cpi) : surface exploitée en maïs (en céréales) sur les plateaux par les agriculteurs de la commune $i ; \mathrm{M} p(\mathrm{C} p)$ : surface totale exploitée en maïs (en céréales) sur les plateaux.
Pour les autres communes, on suppose que les agriculteurs implantent indifféremment leurs cultures sur les plateaux et dans les plaines, sans installer de prairies sur les plateaux, soit :

$$
\mathrm{M} p i=\mathrm{M} i \times \mathrm{T} p i / \mathrm{T} l i \text { et } \mathrm{C} p i=\mathrm{C} i \times \mathrm{T} p i / \mathrm{T} l i
$$

La somme des surfaces en maïs exploitées par les agriculteurs de chaque commune sur les plateaux donne la surface totale en maïs sur ces derniers. Il en est de même pour les surfaces en céréales.

$$
\sum \mathrm{M} p i=\mathrm{M} p \text { et } \sum \mathrm{C} p i=\mathrm{C} p
$$

L'ensemble de ces relations permet d'estimer les surfaces en maïs (respectivement en céréales) des plateaux pour les années 1955, 1970, 1979 et 1988. Celles des années intermédiaires sont déduites, en supposant une évolution linéaire, par interpolation linéaire entre 1955 et 1970 , entre 1970 et 1979 , et entre 1979 et 1988 , sauf pour le maïs dont la production est considérée comme nulle avant 1970.

\section{Estimation des apports en produits phytosanitaires}

Les quantités de produits phytosanitaires utilisées sur les plateaux ont été estimées par deux méthodes d'enquêtes.

La première consiste en une enquête auprès de l'ensemble des agriculteurs du secteur considéré (30) sur leurs pratiques de protection des cultures, afin de chiffrer avec un maximum de précision les quantités de produits phytosanitaires apportées sur l'ensemble des parcelles du territoire étudié. Les limites de cette technique, utilisée par Schiavon et al (1990), sont liées au taux de réponse des agriculteurs ainsi qu'à la validité de leurs dires.

La deuxième méthode repose sur l'interrogation des techniciens locaux, vendeurs de produits phytosanitaires (deux, dont un travaillant pour une société privée et l'autre pour une coopérative agricole). Elle a pour objectif de connaître les programmes de traitements conseillés aux agriculteurs, ainsi que d'avoir une description du comportement de ces derniers face à l'emploi des phytosanitaires. Au cours de ces entretiens semi-directifs, nous tentons aussi de connaître les quantités effectivement vendues.

\section{RÉSULTATS ET DISCUSSION}

\section{Évolution de l'occupation du sol}

Les résultats obtenus avec les différentes méthodes, présentés par le tableau I, sont exprimés en pourcentage de la surface agricole utile de la zone étudiée.

La similitude des résultats issus de l'interprétation des images aériennes et des observations de 
terrain pour les années 1993 et 1994 permet de supposer que ces deux techniques d'évaluation des surfaces en maiis et en céréales sont équivalentes.

Par contre les données brutes issues des RGA communaux des années 1979 et 1988 sont en totale discordance avec les résultats provenant de l'interprétation des images aériennes pour ces mêmes années. Par conséquent, si l'on considère que les résultats obtenus par l'interprétation des images aériennes sont fiables, il convient de rejeter ceux obtenus avec les données brutes du RGA.

Mais, après modification selon la méthode de correction proposée (RGAm), les données obtenues concordent à plus de $98 \%$ avec les interprétations des images aériennes. Les hypothèses de cette méthode ont d'ailleurs été confirmées par les agriculteurs.

Finalement, dans notre cas, l'évolution de l'occupation du sol depuis 1955 peut être estimée à l'aide de l'observation de terrain, du traitement de photographies aériennes et de l'interprétation des données des RGA communaux corrigées selon les caractéristiques du site étudié.

\section{Pratiques phytosanitaires}

L'ensemble des agriculteurs exploitant les terres des huit bassins d'alimentation ont répondu à notre enquête. L'intégralité de la SAU (parcelle par parcelle) a été couverte par leurs réponses pour les années 1989 à 1993. Pour les années antérieures, seules des données concernant l'exploitation du maïs ont pu être collectées. Les matières actives, utilisées à plus de $20 \mathrm{~kg}$ sur l'ensemble de la SAU, présentées par le tableau II avec leurs dates de première utilisation, ainsi que la surface traitée et la dose de traitement depuis 1989.

La comparaison des matières actives et des doses appliquées selon les agriculteurs avec les programmes de traitement fournis par les techniciens (tableau III) montre de fortes différences. Celles-ci s'expliquent par des disparités d'utilisation selon la flore ou les ravageurs réellement présents. En effet, les programmes de traitement indiquent les matières actives à utiliser pour l'ensemble des adventices et ravageurs susceptibles de nuire à la culture. Elles ne sont pas toutes utilisées. Par exemple, les traitements anti-taupins

Tableau I. Évolution de l'assolement selon les quatre méthodes d’évaluation.

\begin{tabular}{|c|c|c|c|c|c|c|c|c|c|c|}
\hline & 1955 & 1970 & 1979 & 1987 & 1988 & 1990 & 1991 & 1992 & 1993 & 1994 \\
\hline \multicolumn{11}{|c|}{ en $\%$ de la $S A U$} \\
\hline \multicolumn{11}{|l|}{ Maïs } \\
\hline $\begin{array}{l}\text { Observation } \\
\text { directe }\end{array}$ & & & & & & 27 & 27 & 28 & 30 & 30 \\
\hline $\begin{array}{l}\text { Photographies } \\
\text { aériennes }\end{array}$ & & & 14 & 26 & 27 & & & & 30 & 30 \\
\hline RGA. & () & 0.5 & 4 & 7 & 8 & & & & & \\
\hline RGAm & () & 1 & 13 & 23 & 27 & & & & & \\
\hline \multicolumn{11}{|l|}{ Céréales } \\
\hline $\begin{array}{l}\text { Observation } \\
\text { directe }\end{array}$ & & & & & & 34 & 35 & 40 & 39 & 40 \\
\hline $\begin{array}{l}\text { Photographies } \\
\text { aériennes }\end{array}$ & & & 40 & 38 & 39 & & & & 39 & 40 \\
\hline RGA. & 46 & 13 & 14 & 14 & 14 & & & & & \\
\hline RGAm & 43 & 38 & 41 & 41 & 41 & & & & & \\
\hline \multicolumn{11}{|l|}{ Autres couverts } \\
\hline $\begin{array}{c}\text { Observation } \\
\text { directe }\end{array}$ & & & & & & 39 & 38 & 32 & 31 & 30 \\
\hline $\begin{array}{l}\text { Photographies } \\
\text { aériennes }\end{array}$ & & & 46 & 36 & 34 & & & & 31 & 30 \\
\hline RGA. & 54 & 86.5 & 82 & 79 & 78 & & & & & \\
\hline RGAm & 57 & 61 & 46 & 36 & 32 & & & & & \\
\hline
\end{tabular}


Tableau II. Inventaire des matières actives utilisées à plus de $20 \mathrm{~kg}$ sur l'ensemble de la SAU.

\begin{tabular}{|c|c|c|c|c|c|c|}
\hline \multirow[t]{2}{*}{ Matière active } & \multirow{2}{*}{$\begin{array}{c}\text { Date de } \\
\text { première } \\
\text { utilisation }\end{array}$} & \multicolumn{5}{|c|}{ Surface traitée (ha) et dose de traitement $(\mathrm{kg} / \mathrm{ha})$} \\
\hline & & Année & Surface & $\begin{array}{l}\text { Dose } \\
\text { minimale }\end{array}$ & $\begin{array}{c}\text { Dose la } \\
\text { plus fréquente }\end{array}$ & $\begin{array}{l}\text { Dose } \\
\text { maximale }\end{array}$ \\
\hline \multirow{5}{*}{$\begin{array}{l}\text { Atrazine } \\
\text { (maïs) }\end{array}$} & 1970 & 1989 & 200 & 2 & 3 & 6 \\
\hline & & 1990 & 200 & 2 & 2,5 & 5 \\
\hline & & 1991 & 200 & 2 & 2,3 & 4 \\
\hline & & 1992 & 200 & 1,5 & 1,7 & 3 \\
\hline & & 1993 & 200 & 1,2 & 1,5 & 2 \\
\hline \multirow{5}{*}{$\begin{array}{l}\text { Pyridate } \\
\text { (maïs) }\end{array}$} & 1989 & 1989 & 2 & 0,8 & 0,9 & 1 \\
\hline & & 1990 & 26 & 0,8 & 0,9 & 1 \\
\hline & & 1991 & 94 & 0,6 & 0,9 & 1 \\
\hline & & 1992 & 158 & 0,5 & 0,9 & 1 \\
\hline & & 1993 & 157 & 0,5 & 0,9 & 0,9 \\
\hline \multirow{3}{*}{$\begin{array}{r}\text { 2,4-MCPA } \\
\text { (céréales) }\end{array}$} & 1991 & 1991 & 44 & & 0,4 & \\
\hline & & 1992 & 56 & & 0,7 & \\
\hline & & 1993 & 107 & & 0,5 & \\
\hline \multirow{3}{*}{$\begin{array}{l}\text { Dichlorprop } \\
\text { (céréales) }\end{array}$} & 1991 & 1991 & 10 & & 0,7 & \\
\hline & & 1992 & 18 & & 0,7 & \\
\hline & & 1993 & 58 & & 1,4 & \\
\hline \multirow{5}{*}{$\begin{array}{l}\text { Isoproturon } \\
\text { (céréales) }\end{array}$} & $<1989$ & 1989 & 0 & & 1,5 & \\
\hline & & 1990 & 6 & & 1,5 & \\
\hline & & 1991 & 15 & & 1,5 & \\
\hline & & 1992 & 36 & & 1,5 & \\
\hline & & 1993 & 6 & & 1,5 & \\
\hline \multirow{5}{*}{$\begin{array}{l}\text { Mécoprop } \\
\text { (céréales) }\end{array}$} & $<1989$ & 1989 & 30 & & 0,5 & \\
\hline & & 1990 & 43 & & 0,6 & \\
\hline & & 1991 & 71 & & 0,9 & \\
\hline & & 1992 & 82 & & 1 & \\
\hline & & 1993 & 148 & & 0,9 & \\
\hline $\begin{array}{l}\text { Prosulfocarbe } \\
\text { (céréales) }\end{array}$ & 1993 & 1993 & 19 & & 4 & \\
\hline
\end{tabular}

sont conseillés pour l'ensemble de la surface en maïs (carbofuran ou terbuphos) et ne sont réalisés en moyenne que sur $10 \%$ des parcelles en maïs. Par conséquent, la seule utilisation des programmes de traitement conseillés pour évaluer les quantités de produits phytosanitaires appliqués sur un territoire donné se traduit par des surestimations qualitatives et quantitatives des matières actives réellement épandues.

Faute de données fournies par les techniciens vendeurs de produits phytosanitaires, il ne nous a pas été possible de comparer les quantités vendues aux agriculteurs à celles déclarées comme appliquées par ces derniers. S'ils ne permettent pas de confirmer les doses appliquées par chaque agriculteur, les entretiens avec les techniciens vendeurs de produits phytosanitaires confirment cependant la nature des produits utilisés et renseignent sur les doses moyennes utilisées. Cette confirmation du tableau II nous permet de supposer que les résultats obtenus par enquête de l'ensemble des agriculteurs sur les quantités réellement appliquées sur le territoire étudié sont valides.

L'enquête agricole a permis de recenser 34 matières actives utilisées sur les 700 ha cultivés.

Depuis 1989, sept d'entre elles ont fait l'objet d'au moins un emploi annuel supérieur à $20 \mathrm{~kg}$ sur l'ensemble de la SAU (fig 1). Ce sont tous des herbicides. Quantitativement, les produits dominants sont l'atrazine, le pyridate et le mécoprop. 
Tableau III. Programmes de traitements conseillés sur maïs et matières actives employées.

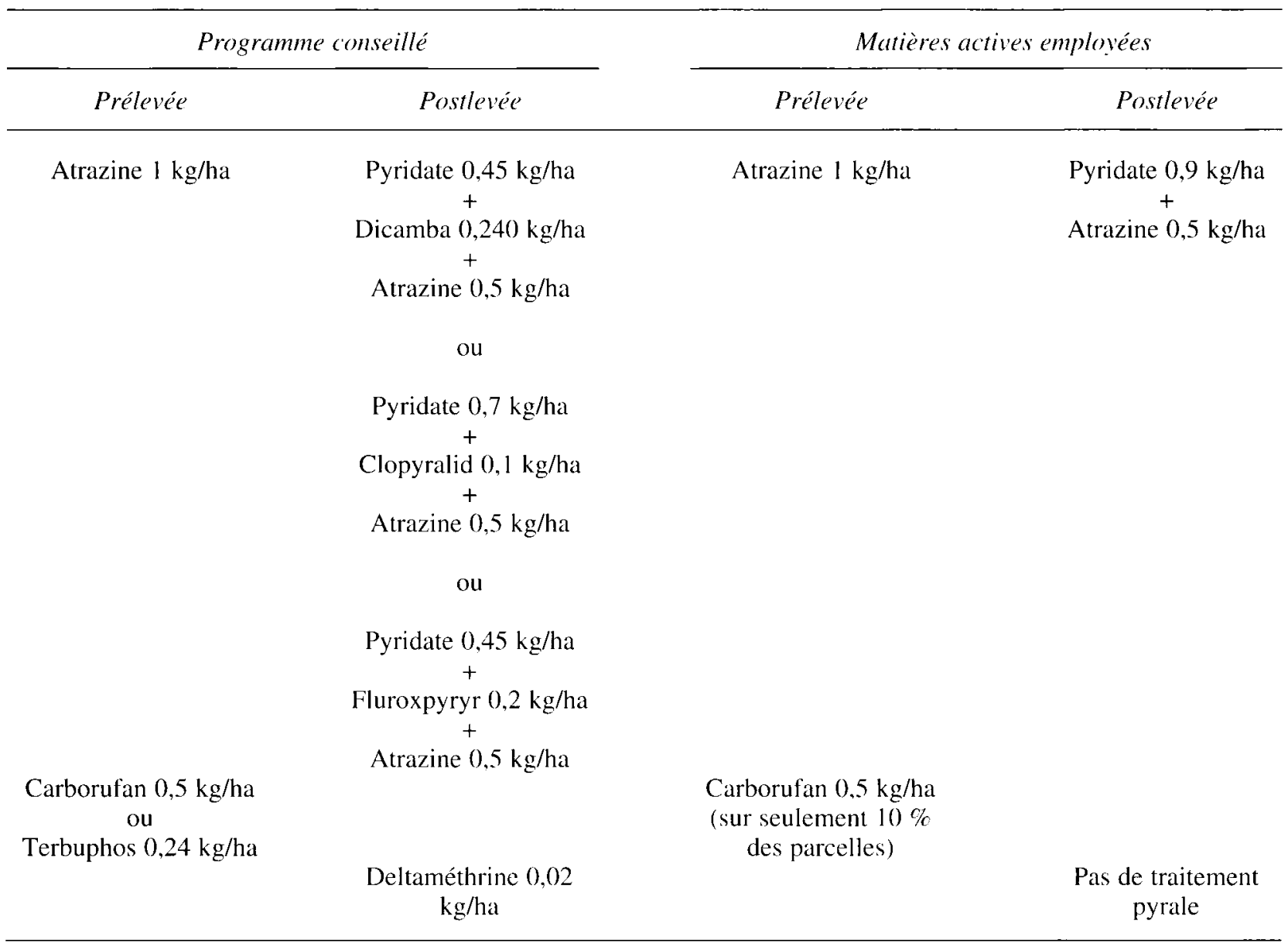

L'atrazine apparaît comme la principale matière active employée depuis 1970. Ses doses d'utilisation ont été divisées par deux depuis 1989 (tableau II). Cette diminution s'est accompagnée de l'apparition de matières actives dont les spectres d'action sont complémentaires de celui de l'atrazine.

Si en 1988, aucune parcelle en maïs ne recevait d'autres matières actives destinées au désherbage, en 1992, $90 \%$ des parcelles recevaient au moins un traitement complémentaire, essentiellement réalisé avec du pyridate.

En moins de 25 ans, environ sept tonnes d'atrazine ont été appliquées sur les 700 hectares cultivés des plateaux du haut Saintois (fig 2). Ainsi, en tenant compte de la rotation des cultures, chaque hectare cultivé a reçu en moyenne $10 \mathrm{~kg}$ d'atrazine en 25 ans, soit $400 \mathrm{~g}$ par hectare et par an. En ce qui concerne le pyridate, dont l'utilisation est en pleine expansion (tableau II), environ $500 \mathrm{~kg}$ de matière active ont été apportés de 1989 à 1993 sur les 200 ha de maïs. On observe une évolution inverse de l'utilisation de l'atrazine et du pyridate. En effet, la diminution de l'utilisation de l'atrazi- ne est compensée quantitativement par celle du pyridate. Ce transfert d'utilisation de l'atrazine vers le pyridate pourrait éventuellement limiter les risques de pollution des eaux. Car, d'après Nigitz et al (1992), le pyridate et son principe actif le CL9673 ne seraient pas susceptibles d'atteindre les eaux souterraines.

Sur la sole céréalière, 28 matières actives ont été employées de 1989 à 1993 dont 17 herbicides et 11 fongicides. Cette diversité s'explique par l'utilisation de produits commerciaux, comprenant jusqu'à quatre matières actives. Le désherbage des céréales n'est pas systématique, mais la proportion de parcelles désherbées est en forte augmentation ( $15 \%$ en 1989 contre $86 \%$ en 1993). Le mécoprop est la molécule la plus employée à la fois en surface et en quantité (environ $450 \mathrm{~kg}$ de 1989 à 1993). Trois autres matières actives ont été apportées à plus de $100 \mathrm{~kg}$ sur cette même période : le dichlorprop, le 2,4-MCPA et l'isoproturon. Les doses moyennes d'application varient d'une année à l'autre car ces matières actives entrent à des concentrations inégales dans la composition des produits commerciaux (tableau II). 
Fig 1. Quantités de matières actives apportées sur les 700 ha cultivés du site depuis 1989 .
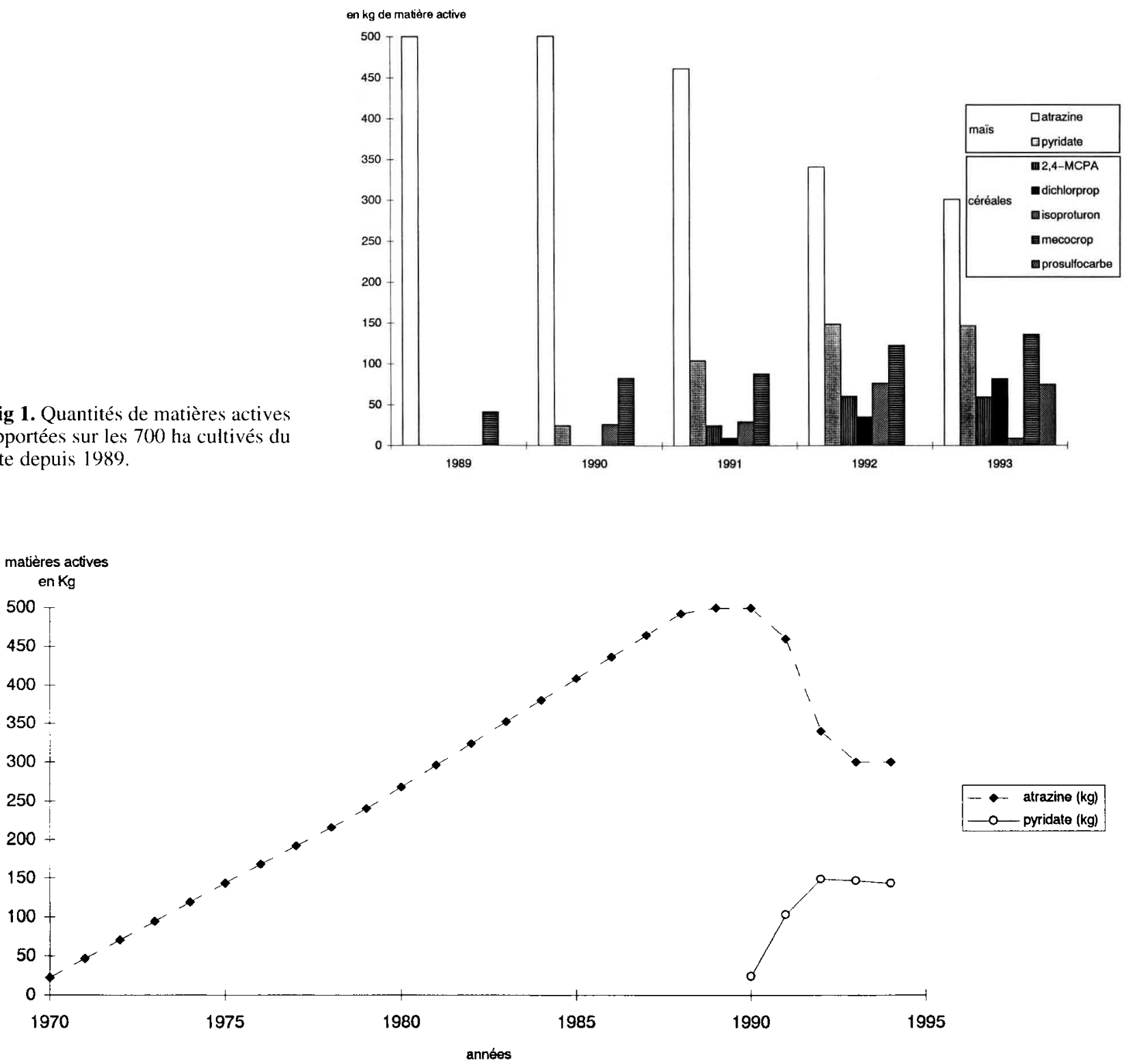

Fig 2. Quantités appliquées annuellement d'atrazine et de pyridate sur les plateaux du haut Saintois de 1970 à 1994

\section{Modalités d'extrapolation}

\section{Reconstitution de l'assolement}

L'assolement d'un territoire peut être considéré comme une variable de forçage des modèles de transfert de polluants d'origine agricole. En effet, si l'on associe une pratique moyenne à une culture, c'est la répartition des cultures sur le territoire qui détermine l'impact total de la pratique. Établir un historique de l'assolement permet dans la majorité des cas d'expliquer l'apparition de l'ensemble des pollutions d'origine agricole. C'est avec l'extension de la culture du maïs que se sont posés les premiers problèmes de pollution de l'eau par l'atrazine et ses métabolites.

Les huit bassins d'alimentation que nous avons étudiés se caractérisent par des assolements homogènes dans leur ensemble, mais fortement contrastés par rapport à la région environnante. L'interprétation des données du RGA peut dans ce cas compléter le traitement des photographies aériennes qui sont les seules données disponibles concernant l'utilisation du territoire dans un passé plus ou moins récent. Cette interprétation des données du RGA sera d'autant plus indispensable lorsque les photographies aériennes ne permettent pas de distinguer toutes les cultures. Ce qui peut être le cas avec des photographies aériennes esti- 
vales. En effet, les céréales et le colza arrivant à maturité à la même époque, ils peuvent donc être tous deux moissonnés lors de la prise de vue.

À l'avenir, l'augmentation probable de la taille des parcelles et l'amélioration de la résolution spatiale des capteurs satellitaires permettront l'utilisation sans restriction des images satellitaires, les données du RGA devenant obsolètes en raison du nombre de plus en plus restreint d'exploitations par commune. La comparaison des résultats obtenus par l'imagerie aérienne ou satellitaire avec des observations visuelles des couverts végétaux réalisées sur le terrain permettra une vérification des résultats et un calage des méthodes de traitement des images.

\section{Quantification des apports phytosanitaires}

En ce qui concerne l'utilisation des produits phytosanitaires sur chaque culture, seule l'enquête réalisée auprès de l'ensemble des agriculteurs permet d'évaluer les quantités apportées. De telles enquêtes posent le problème de la fiabilité des informations fournies "de mémoire » par les agriculteurs pour les données culturales anciennes, surtout en zone d'élevage. En effet si le cahier parcellaire est d'utilisation assez courante dans les zones de grandes cultures, il est quasiment inexistant chez les éleveurs qui ne notent pas les itinéraires culturaux de leurs parcelles céréalières fourragères.

Les meilleurs résultats sont obtenus pour les cultures traitées avec un nombre restreint de matières actives, comme c'est le cas du maïs traité exclusivement à l'atrazine. Car la diversité des produits ne permet généralement pas à l'agriculteur de se remémorer l'ensemble des produits et des quantités appliqués.

Cette méthode se limite à l'estimation des apports d'origine agricole. Pour les territoires dont l'activité n'est pas exclusivement agricole, il convient de tenir compte de l'ensemble des utilisations non agricoles.

\section{CONCLUSIONS}

L'observation de terrain, le traitement de photographies aériennes ou l'interprétation des données du RGA permettent d'instruire l'évolution de l'occupation du sol des plateaux du haut Saintois, depuis 1955. Pour les années où elles ont pu être utilisées simultanément, les différentes méthodes apparaissent équivalentes. Elles sont complémentaires pour les autres années. Cette démarche peut être reconduite dans le cas de territoires homogènes présentant de forts contrastes avec les régions voisines. Dans les autres cas, l'utilisation conjointe de l'imagerie aérienne ou satellitaire et des observations de terrain devrait permettre la reconstitution de l'assolement.

L'enquête semi-directive auprès de l'ensemble des agriculteurs exploitant ces territoires semble un moyen efficace pour déterminer les tendances d'application des différentes spécialités commerciales sur chaque culture.

Finalement, la démarche présentée fournit des renseignements concernant les apports de produits phytosanitaires dans les sols d'une région agricole. Néanmoins, elle ne permet pas de les chiffrer avec exactitude. Elle est cependant essentielle dans l'estimation des éventuelles contaminations des sols agricoles et des eaux destinées à la consommation humaine par les résidus de produits phytosanitaires, car tous les modèles de transfert mis au point ou en cours d'élaboration nécessite ce paramètre clé. Enfin, l'extension de cette démarche à d'autres régions présentant des risques de pollution est subordonnée au choix d'une méthode d'évaluation de l'assolement adaptée à cette région.

\section{RÉFÉRENCES}

Domagalsky JL, Dubrovsky NM (1992) Pesticide residues in groundwater of the San Joaquim Valley, California. J Hydrology 130, 299-338

Gaury F (1992) Systèmes de culture et teneurs en nitrates des eaux souterraines. Thèse de docteuringénieur, sciences agronomiques, Ensa Rennes, $229 \mathrm{p}+$ annexes

Girard MC, Girard CM (1989) Télédétection appliquée : zones tempérées et intertropicales. Masson, collection «Sciences agronomiques », Paris, $255 \mathrm{p}$

Goolsby DA, Thurman EM, Kolpin DW (1991) Herbicides in streams: Midwestern United States. In : Irrigation and Drainage Proc, Honolulu, 22-29, July 1991

Heydel L, Benoît M, Schiavon M (1994) Qualité des caux et pratiques phytosanitaires agricoles, XXIV Congrès du groupe français des pesticides, Bordeaux, 18-19 mai 1994, 111-116

Heydel L, Schiavon M, Benoît M (1996) Transfert de produits phytosanitaires à l'échelle du bassin d'alimentation, $5^{\mathrm{es}}$ Journées nationales de l'étude des sols, Rennes, 22-25 avril 1996, $115-116$ 
Hörmann WD, Tournayre JC, Egli H (1979) Triazine herbicide residues in central european streams. Pestic Monitor J 13, 128-131

Knisel WG (1980) CREAMS: A field-scale model for chemicals, runoff, and erosion from agricultural management systems. Conservation Research Report $\mathbf{n}^{\circ} 26$, US Department of Agriculture, Washington, DC, $643 \mathrm{p}$

Knisel WG (1993) GLEAMS: Groundwater Loading Effects of Agricultural Management Systems. Version 2.10 Tifton GA, University of Georgia, Publication $n^{\circ} 5$

Maupas M, Gillet M (1992) Premier bilan de la contamination de quelques rivières de Bretagne par les produits phytosanitaires. Colloque Phyteau : Eau Produits phytosanitaires - Usages agricoles et connexes, Versailles, 21-22 octobre 1992, 13-18

Morange H, Salleron JL (1982) Étude des apports d'azote et de phosphore dans les rivières du bassin de la Moselle. Agence de l'eau Rhin-Meuse, Moulins-les-Metz, $111 \mathrm{p}+$ annexes

Mouvet MM, Dousset S, Landreau A (1992) Combinaison d'approches pour l'étude des transferts de pesticides depuis la surface du sol jusqu'aux eaux souterraines. Colloque PHYTEAU : Eau - Produits phytosanitaires - Usages agricoles et connexes, Versailles 21-22 octobre 1992, 81-86

Nigitz HP, Zohner A, Gilbert E (1992) Comportement du pyridate dans le sol et l'eau : méthodes d'études et résultats. Colloque PHYTEAU : Eau - Produits phytosanitaires - Usages agricoles et connexes, Versailles 21-22 octobre 1992, 113-120

Pionke HB, Glotfelty DW (1989) Nature and extent of groundwater contamination by pesticides in agricultural watershed. Wat Res. 23, 1031-1037

Salou MC (1992) Maîtrise de la qualité des eaux en milieu agricole. Exemple des plateaux calcaires de Vicherey et d'Aboncourt. Mémoire DEA, université de Metz, Inst nat rech agron syst agr dev, Mirecourt, $126 p+$ annexes

Schiavon M, Thévenot X, Andreux F, Portal JM (1990) Relation entre traitements phytosanitaires et contamination des captages. Approche méthodologique sur quelques bassins d'alimentation de Lorraine, Drass de Lorraine, $72 \mathrm{p}+$ annexes 